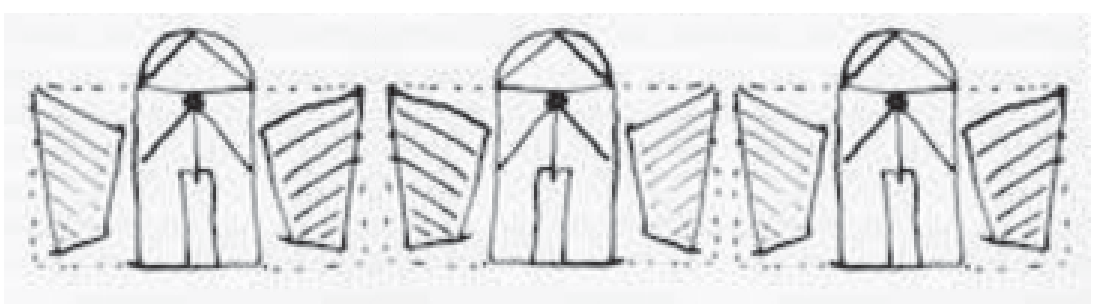

\title{
Antropologia nos serviços de saúde: integralidade, cultura e comunicação
}

Francisco Arsego de Oliveira ${ }^{1}$

OLIVEIRA, F. A. Anthropology in healthcare services: integrality, culture and communication, Interface _ Comunic, Saúde, Educ, v.6, n.10, p.63-74, 2002.

The use of other fields of knowledge in the healthcare area has intensified over the last few years. Social Sciences in general and specifically Medical Anthropology have contributed decisively to the understanding of phenomena related to the health/disease process, both individually and collectively. In this paper, the author discusses a number of considerations, in order to reach a better understanding of the issue of providing healthcare, focusing on clearly revealing the forms of interaction and communication between these healthcare services and their users. The article concludes by showing how centering medical practice on users and their culture ends up being beneficial to both patients and professionals, in that it recovers the humane aspects and the holistic focus of healthcare.

KEY WORDS: Medical anthropology; healthcare services; health assistance.

A utilização de outras áreas do conhecimento no campo da saúde tem sido intensificada nos últimos anos. As Ciências Sociais de modo geral e a Antropologia Médica, especificamente, têm contribuído de maneira decisiva para a compreensão dos fenômenos relacionados ao processo saúde/doença, tanto individual como coletivamente. Neste artigo, o autor tece uma série de considerações buscando um melhor entendimento para a questão do atendimento de saúde, com ênfase na explicitação das formas de interação e comunicação entre esses serviços de saúde e seus usuários. $\mathrm{O}$ artigo conclui mostrando como o fato de centrar a prática médica no usuário e na sua cultura acaba trazendo benefícios para pacientes e profissionais, no sentido do resgate da humanização do cuidado e da integralidade da atenção à saúde.

PALAVRAS-CHAVE: Antropologia médica; serviços de saúde; assistência à saúde.

${ }_{1}^{1}$ Professor do Departamento de Medicina Social, Faculdade de Medicina, Universidade Federal do Rio Grande do Sul/UFRGS; Médico do Serviço de Saúde Comunitária do Grupo Hospitalar Conceição, Porto Alegre, RS. <arsego@via-rs.net> 


\section{Introdução}

Muito tem sido falado e escrito sobre o cuidado à saúde no Brasil e no mundo nos últimos tempos. Além da literatura especializada sobre o tema, praticamente todos os dias deparamo-nos com matérias em jornais, revistas, rádio, televisão e mesmo em conversas informais, sobre casos de pessoas que não foram atendidas, pessoas que morreram, tiveram seqüelas pela falta de atendimento médico ou mesmo pessoas que foram mal atendidas e até as que foram atendidas e não tiveram seus problemas resolvidos. Restringindonos ao caso brasileiro, é bem verdade que nem sempre se fala só do caos do sistema de saúde e dos absurdos que ocorrem em função dele. Há novas descobertas sendo feitas, novos medicamentos sendo desenvolvidos, mecanismos de doenças sendo elucidados, cirurgias revolucionárias, aparelhos fantásticos, "dicas" para uma "vida saudável" que nos inundam de informações. O que não deixa de ser um quadro paradoxal: grandes $e$ incontestáveis avanços tecnológicos em benefício do ser humano, por um lado, e, por outro, uma sensação de crise permanente, com atendimento inadequado, insuficiente e, pior, oferecido sem eqüidade.

A preocupação com a saúde definitivamente incorporou-se ao nosso cotidiano. Só que, ainda hoje, para perplexidade de alguns, nem sempre todos os problemas de saúde são vistos dentro do sistema formal de cuidado à saúde. Pelo contrário: calcula-se que 70 a $90 \%$ dos episódios de doença são manejados fora desse sistema, por autocuidado ou busca de formas alternativas de cura (Kleinman et al., 1978; Kleinman, 1980; Knauth, 1991). Ou seja, o modelo biomédico é apenas um entre tantos sistemas disponíveis no "mercado" da saúde. O que há de comum entre esses diversos sistemas e que gostaríamos de explorar um pouco mais neste artigo é o encontro que se estabelece entre o paciente e o agente de cura ${ }^{2}$. Tal encontro possui elementos peculiares referentes à comunicação, freqüentemente negligenciados na prática médica e de saúde em geral (Helman, 1994; Pedersen \& Baruffati, 1989). Mais do que isso, essas peculiaridades são moldadas de maneira marcante pelas características culturais de cada grupo social envolvido.

\section{Interações entre serviços de saúde e usuários}

Mesmo sob o ponto de vista formal, a relação entre serviços de saúde e usuários envolve muitos outros aspectos além do encontro físico entre médico e paciente num consultório, por exemplo. Há, entre outros fatores, as políticas de saúde em cada local e as concepções dos indivíduos sobre o que é estar doente. A doença é uma experiência que não se limita à alteração biológica pura, mas esta lhe serve como substrato para uma construção cultural, num processo que lhe é concomitante. Não queremos dizer com isso que exista uma seqüência de "primeiro biologia e depois cultura", mas sim que existem percepções culturais acerca de um fenômeno que também abarca o biológico, mas que o supera. Ou seja, uma determinada pneumonia bacteriana pode ser causada pelo mesmo agente infeccioso em todo o mundo, com alterações fisiopatológicas equivalentes

\footnotetext{
${ }^{2} \mathrm{Na}$ falta de uma tradução melhor, "agente de cura" é utilizado no sentido do termo healer, em inglês, e que pode ser o médico, o curandeiro, o erveiro, o pai-de-santo ou outro profissional da saúde que desempenhe essa função.
} 
em diversos indivíduos. Mas a forma de tratamento, o sistema de saúde disponível e, sobretudo, a percepção que a pessoa acometida terá sobre a sua doença variarão enormemente.

Isso fica mais claro quando conseguimos estabelecer a diferença agora já clássica entre illness e disease (Kleinman et al., 1978; Kleinman, 1980; Kleinman, 1986). E aqui nos permitimos o uso dos termos em inglês por terem um sentido mais acurado, uma vez que, em português, a palavra "doença" contempla os dois significados.

Podemos dizer que illness, o equivalente a "perturbação", é a forma como os indivíduos e os membros de sua rede social percebem os sintomas, categorizam e dão atributos a esses sintomas, experenciando-os, articulando esse sentimento por meio de formas próprias de comportamento $e$ percorrendo caminhos específicos em busca da cura. Além da experiência pessoal, o indivíduo atribui significado à doença. Enfim, illness é a resposta subjetiva do indivíduo à situação de doença, uma resposta que engloba aspectos individuais, sociais e culturais à experiência de estar doente.

Por outro lado, disease é a forma como a experiência da doença (illness) é reinterpretada pelos profissionais de saúde à luz de seus modelos teóricos e que os orienta em seu trabalho clínico. É portanto, uma definição de disfunção, assentada num substrato essencialmente biomédico. E aqui, por biomedicina, referimo-nos à teoria e prática médica predominante no ocidente e amplamente disseminada em todo o mundo. Tem como sinônimo expressões que, em geral, definem a Medicina como "ocidental", "científica" e "alopática" e apresenta entre suas principais características o foco sobre o ser humano enquanto entidade essencialmente biológica (Hahn \& Kleinman 1983; Williams \& Calnan, 1996). Uma das atribuições principais do médico é, então, "traduzir" o discurso, os sinais e os sintomas do paciente para chegar ao diagnóstico da doença, ou seja, decodificar illness em disease.

Assim, como nos referimos a uma relação que se estabelece entre serviços e usuários, isso pressupõe uma comunicação com duas vias de fluxo, permitindo, no momento em que o indivíduo busca atendimento de saúde, o encontro de duas visões de mundo diferentes, entre illness, por um lado, $e$ disease, por outro. Restringindo-nos aos serviços formais, é importante que se diga que esse encontro, como já foi frisado acima, não se resume apenas à consulta médica, mas se dá também na realização de exames, na administração de um medicamento, no momento em que um indivíduo freqüenta uma atividade em grupo no posto de saúde etc. Quando uma pessoa procura um determinado serviço de saúde, ela lança mão de uma série de mecanismos que orientam tal procura e que são acionados muito antes do encontro propriamente dito: quais os sintomas que a forçam ao encontro, qual o momento adequado de fazê-lo, qual o profissional buscado, qual o linguajar a ser utilizado para descrever ao médico o que sente, que a roupa vestir na consulta, e assim por diante. Em última análise, como é que o indivíduo se prepara para esse momento, como se comporta nele e qual a "bagagem" que carrega consigo? Portanto, quando mencionamos "comunicação", também queremos dizer que há produção de significados de ambos os lados e que a cultura de cada grupo social estará igualmente sempre presente. 
Buscando abordar esse assunto entre profissionais da saúde utilizamos um recurso figurativo da anatomia humana, que acabamos chamando jocosamente de uma "teoria peritonial da cultura". Isso porque, embriologicamente, na formação do abdômen, há a membrana peritonial (ou peritônio) que recobre internamente toda a cavidade como um balão inflado (Langman, 1977). Essa membrana, ao recobrir as vísceras, tem a finalidade de defendê-las, ao mesmo tempo em que facilita o deslizamento dos vários órgãos, fornece alimento e permite a absorção de líquidos. No desenvolvimento do embrião, os órgãos abdominais, como que se originando dessa parede, ao projetarem-se para o interior da cavidade, carregam consigo essa mesma membrana, como se fosse o dedo de uma luva (figura 1).

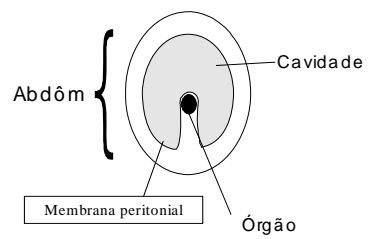

FIGURA 1. Formação esquemática do peritônio, a partir de Langman (1977)

O peritônio, uma membrana que é semipermeável, permite, portanto, trocas de substâncias através dele. De modo análogo, podemos então dizer que, no cenário de um serviço de saúde, há pelo menos dois "dedos de luva" se encontrando, recobertos por uma hipotética "fina membrana semipermeável": a do profissional de saúde e a do paciente, cada um deles conectado, no momento do contato, ao seu universo cultural e simbólico, que o "alimenta", dá sustentação e possibilita a realização de "trocas" (figura 2).

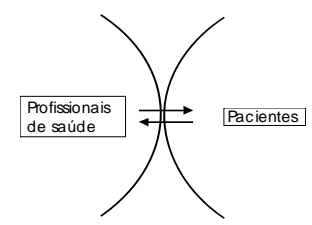

FIGURA 2. Encontro entre profissionais e pacientes, cada um recoberto por uma "membrana da sua cultura"

Analisando o fenômeno sob esse prisma, uma das questões centrais ao defrontar médicos e pacientes é o encontro de modelos explanatórios (ou explicativos) diferenciados, o que foi bem desenvolvido nos trabalhos de Kleinman et al. (1978) e Kleinman (1980). Segundo esses autores, a experiência da doença é moldada culturalmente, o que determina a maneira como percebemos e como buscamos superá-la. Podemos dizer que nós literalmente "aprendemos a ficar doentes", de acordo com o nosso meio social, que influencia diretamente a forma como sentimos as doenças, expressamos seus sintomas e utilizamos os recursos de cura à nossa disposição. Há, resumidamente, pelo menos cinco elementos envolvidos nesse modelo explanatório sobre a doença: 1) etiologia do problema; 2) duração $e$ 
características dos sinais e sintomas iniciais; 3) fisiopatologia do problema; 4) evolução natural e prognóstico; e 5) tratamento indicado para o problema. Ou seja, utilizando o exemplo da pneumonia citado anteriormente, poderíamos dizer que, quando ficamos doentes, procuramos identificar a causa do que nos aflige ("isso é gripe mal curada", "foi praga de alguém" ou ainda "peguei uma friagem"); analisamos o que estamos sentindo ("febre, calafrios, tosse"); buscamos compreender quais os mecanismos fisiológicos ("o pulmão está ruim, cheio de catarro", "meu pulmão está fraco por causa do cigarro"); fazemos uma análise da sua gravidade ("posso morrer disso?"); e, por fim, buscamos estabelecer um plano para que possamos retornar à situação anterior, sem a doença ("bom para curar isso é uma injeção", "vou tomar um passe").

Este modelo e estes elementos nem sempre são pensados nessa seqüência ou estão completamente articulados entre si e podem apresentar inconsistências, erros e, até mesmo, contradições internas. Mas é a base a que o paciente recorre para tentar dar coerência ao que está ocorrendo com ele na situação de uma doença específica. O que deve ficar claro é que esse pensamento segue uma lógica diferente daquela do modelo utilizado pelo médico.

Quando, então, esses diferentes modelos se encontram no momento da consulta ou do contato com o serviço de saúde, a saída possível para que o processo clínico tenha sucesso é uma "negociação entre as partes", nem sempre totalmente consciente, cada uma usando os seus argumentos para que se chegue a um consenso possível para aquele momento. Ou seja, deve haver uma concordância, mesmo que temporária, entre o agente de cura (o médico, por exemplo) e o paciente em cada um dos itens acima. E para que o sucesso desejado seja atingido, além dos modelos explanatórios tornarem-se ao menos parcialmente manifestos, também deve haver uma postura receptiva à negociação, de parte a parte.

É interessante perceber que, apesar de a perspectiva antropológica vir auxiliando no entendimento desses fenômenos cada vez com mais clareza $e$ veemência, o modelo biomédico é ainda brutalmente hegemônico, o que tem levado a uma visão reducionista da doença, vista como processo exclusivamente biológico.

Mas a contribuição dos antropólogos médicos às questões da saúde não se limita apenas à noção do modelo explanatório. Ela avança no sentido de explicitar que todas as atividades relacionadas com o cuidado à saúde estão interrelacionadas, tendendo a constituir uma forma socialmente organizada para enfrentar a doença, e formam, a exemplo da religião e da linguagem, um sistema cultural próprio, que é o sistema de atenção à saúde (Kleinman, 1980; Rhodes, 1996). Em cada cultura, a doença, a resposta a ela, os indivíduos que a experienciam, os que se ocupam em tratá-la e as instituições envolvidas estão interconectados mediante esse sistema, que também contempla, entre outros elementos, as crenças sobre a origem das doenças, as formas de busca e avaliação do tratamento, os papéis desempenhados $e$ as relações de poder entre todos os envolvidos (Calnan, 1988; Foucault, 1979; Boltanski, 1984). Os pacientes e os agentes de cura são componentes básicos do sistema, estando imersos num contexto de 
significados culturais e de relações, não podendo ser entendidos fora dele (Bodstein, 1995).

Tanto as crenças quanto os padrões de comportamento dos indivíduos fazem parte desse sistema de atenção à saúde e são, em grande parte, derivados de regras culturais. Neste sentido, a inclusão do pensamento de Geertz (1978) torna-se pertinente nesta discussão, pois esse autor defende a idéia da cultura como uma "teia de significados" e da importância de a analisarmos enquanto ação e como sistema simbólico. Como ação, a forma de expressão da cultura é pública, pressupondo, no discurso social, a existência de "protagonistas" e "assistentes" que se comunicam entre si a partir de interpretações de códigos socialmente estabelecidos de modo prévio. Ou seja, como diz o autor, "a cultura é pública porque o significado o é". Isso é importante especialmente em relação ao tema deste artigo, uma vez que parece ser evidente que os aspectos relacionados à saúde/doença envolvem ações dos indivíduos, expressos na forma como percebem a doença, estabelecem um "diagnóstico" e buscam um tratamento.

A outra contribuição importante de Geertz é quanto ao próprio método para desvendar a cultura e seus significados, que é, segundo o autor, essencialmente interpretativo. O trabalho etnográfico presta-se muito bem para isso, ao tentar "ler" o que ocorre à sua frente, decifrando não apenas o que está explícito, à superfície, mas também os comportamentos aparentemente "incoerentes" e "deslocados", que, em última análise, têm respaldo na realidade simbólica de nossos pacientes. Em outras palavras, é a realidade simbólica que permitiria ao paciente atribuir significados a partir de sua experiência individual e segundo as normas sociais e culturais do seu grupo.

E é por essa razão que, como qualquer outro sistema cultural, precisa ser entendido em termos de sua atividade instrumental e simbólica. Como afirma Kleinman (1980), estudos de nossa própria sociedade e investigações comparativas devem iniciar contemplando a atenção à saúde como um sistema que é social e cultural na sua origem, estrutura, função e significado.

E por isso é também importante a discussão sobre a construção social da realidade, abordada por Berger \& Luckman (1995), no sentido de tornar claras as relações humanas estabelecidas pelo indivíduo com os outros e com o mundo que o rodeia. São relações governadas por regras culturais específicas, incorporadas pelos indivíduos, que as provêem de significado $e$ as legitimam perante o grupo social.

Esta realidade social não é única no tempo e no espaço, monolítica a ponto de não permitir variações individuais e coletivas. Mas o fundamental é perceber que ela exerce uma influência decisiva na maneira como cada um de nós pensa e age diante de uma situação de doença, optando por um determinado tipo de atendimento e avaliando seu resultado (Atkinson, 1993). Baseado nisso, Kleinman (1980, p.38) sustenta que

(...) a prática clínica (tradicional e moderna) ocorre em e cria mundos sociais particulares. Crenças sobre doenças, os comportamentos exibidos pelas pessoas doentes - inclusive as suas 


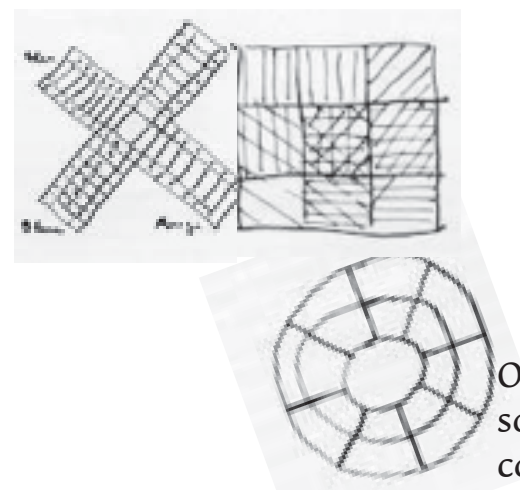

expectativas frente ao tratamento - e o modo como as pessoas doentes reagem em relação à família e aos profissionais de saúde são todos aspectos da realidade social. Elas, como o próprio sistema de saúde, são construções culturais, moldadas distintamente em diferentes sociedades e nos diferentes contextos sociais dentro dessas mesmas sociedades. (nossa tradução)

O mesmo autor avança na sua análise ao denominar os aspectos da realidade social relacionados à saúde de realidade clínica. Esta noção refere-se ao contexto social que influencia o desenvolvimento da doença e o cuidado clínico estabelecendo, assim, uma interseção entre as realidades clínica $e$ simbólica, de um lado, e a realidade física e psicobiológica de outro. Esse conceito é útil sobretudo para evidenciar ainda mais como o fenômeno clínico é também socialmente constituído e, de modo recíproco, como o mundo social pode ser clinicamente construído. Analogamente ao conceito de realidade social, a realidade clínica também está em constante mudança, variando conforme o grupo social. Permite igualmente uma análise em diversos níveis, tanto macro como microssocial, da sociedade como um todo até pequenas "comunidades" isoladas.

O que ocorre, então, é uma relação de cumplicidade entre quem presta $e$ quem recebe o serviço, isto é, o serviço de saúde deve ser aceito socialmente, no sentido de poder ser procurado em caso de doença. Isso não implica, contudo, uma forma homogeneizada de busca de atendimento. O que queremos dizer é que, apesar de o sistema de atenção à saúde ser uma construção coletiva, o padrão de uso do mesmo difere de acordo com o grupo social, com as famílias e mesmo com os indivíduos, dependendo, entre outros fatores, do grau de instrução da pessoa, de sua religião, de sua ocupação, da rede social a que pertence e, concretamente, das doenças existentes.

Esta argumentação de certa forma elucida a questão da uniformidade que supostamente as comunidades teriam nesse aspecto. Assim, apesar de possuírem uma série de elementos em comum, os membros dessas comunidades podem apresentar comportamentos diferentes em relação ao cuidado de saúde. Quem não se lembra na sua prática profissional dos "consultadores freqüentes", dos pacientes que permanecem mais afastados $e$ que consultam exclusivamente nas situações de doença grave, dos que trazem somente os filhos para acompanhamento médico, dos que trazem apenas os idosos, e assim por diante?

Como pode ser observado, a relação que as comunidades estabelecem com os seus serviços de saúde é complexa, pois envolve aspectos culturais de ambos os lados. Ou seja, é uma questão da própria dinâmica da interação, em que está em jogo a legitimação do serviço frente à comunidade, que de certa maneira outorga poder a esse grupo de profissionais para lidar com alguns de seus problemas de saúde.

Mas, como vimos no início deste artigo, o sistema de atenção à saúde não se limita ao setor formal. Kleinman (1980) sustenta que esse sistema é composto, de modo genérico, por três partes que por vezes se sobrepõem: o setor popular, o setor profissional e o setor folk, que pode ser traduzido 
como setor paraprofissional (figura 3).

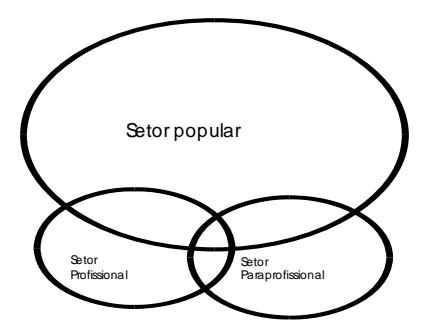

FIGURA 3. Sistema de atenção à saúde, adaptado de Kleinman (1980)

O setor popular, em geral, o maior deles, é aquele em que a família e o grupo social mais próximo desempenham papel importante. É um espaço eminentemente "leigo", onde a doença começa a ser definida e onde são desencadeados os vários processos terapêuticos de cura. Já o setor profissional representa a organização formal da prática de saúde, na maior parte dos casos, tendo a Biomedicina como referência. É o setor que, em certos países, por ser mais desenvolvido, organizado e poderoso, acabou submetendo todas as outras práticas de saúde à sua autoridade. Pela sua grande penetração e pelo poder de que dispõe em algumas sociedades, como a brasileira, o setor profissional busca impor-se sobre os demais setores, forçando ao que tem sido referido como um processo de medicalização da sociedade, baseado num modelo "científico" e biológico (Camargo Jr, 1995; Luz, 1979). E por fim, o setor paraprofissional, que abrange todas as demais práticas de saúde "não-profissionalizadas", como erveiros, benzedores, práticas religiosas e outras formas alternativas de cura. Este modelo proposto é conveniente, pois pode ser adaptado a diversas sociedades. Assim, os chamados países desenvolvidos possuem um setor profissional muito vigoroso, ao passo que outros países podem apresentar o setor para-profissional maior. Da mesma forma, esse modelo aplica-se igualmente bem no nível local, onde também os três setores atuam de forma simultânea.

\section{Considerações finais}

Em geral, tem-se a visão de que o atendimento à saúde é organizado pelos técnicos para ser simplesmente "usufruído" pelos pacientes, que assumiriam uma posição um tanto quanto passiva diante do que lhes é ofertado - talvez devêssemos dizer que os serviços de saúde são "equipecêntricos", um tipo particular de etnocentrismo, em que a equipe de saúde passa a julgar seus usuários a partir da visão de seus membros, estabelecendo unilateralmente o que é certo ou errado, adequado ou inadequado em relação ao cuidado à saúde. Convém lembrar, porém, que é esse "leigo" quem faz a escolha final se realiza ou não a consulta, quando vai fazê-lo e onde. Mesmo depois da consulta ter-se consumado, é ainda ele quem detém o poder de cumprir ou não as determinações médicas ou mesmo procurar outras alternativas.

De fato, os pacientes circulam muito livremente entre esses diversos 
setores de atenção à saúde, que na verdade possuem limites não tão estritos, ocorrendo apenas uma mudança de linguagem e postura frente a eles nesse "trânsito" de um setor para o outro.

Este artigo insere-se então nesse esforço da Antropologia, mais especificamente da Antropologia Médica, de compreender a influência da cultura na maneira como os indivíduos percebem a doença e estabelecem relações com os diversos sistemas de saúde.

As reflexões aqui contidas nada mais são do que uma tentativa de repensar nossa postura como profissionais de saúde. Fazendo isso, inevitavelmente estaremos, mesmo que de forma indireta, questionando uma série de outros assuntos bem atuais: o sistema formal de saúde, o Sistema único de Saúde (SUS), o trabalho médico, a cidadania, a alteridade, a democratização dos serviços de saúde e a forma de interação desses com os seus usuários e vice-versa.

Mas o que há de concreto nisso tudo? Nossa primeira convicção refere-se à necessidade de ampliarmos nossos conceitos sobre saúde/doença em nossa atividade clínica, $e$ isso vale para todas as especialidades médicas e de saúde em geral. Apesar do discurso ser fácil, quando chega o momento de sua aplicação na realidade, deparamo-nos com uma prática que, com muita facilidade, expurga os aspectos sociais, políticos, econômicos e culturais do processo saúde/doença.

É hora, portanto, de reconduzirmos o "paciente" ao centro da relação com os serviços de saúde, superando a visão de uma relação médico-paciente tradicional e partindo para uma relação efetiva entre sujeitos, que são diferentes, é claro, mas ainda assim sujeitos. Sem nos anularmos como profissionais da saúde - isso seremos sempre -, somos nós que temos que conhecê-lo melhor, respeitá-lo e permitir adaptações da nossa prática a ele, e não o contrário, como vem acontecendo até agora. Não nos esqueçamos que o objetivo final dos serviços de saúde ainda é o paciente/usuário.

Resgatar a cultura para o centro da relação estabelecida entre indivíduos e os serviços de saúde é um esforço que desencadeia uma série de implicações na forma como esse relacionamento será concretizado. Kleinman (1980) afirma que, no contexto atual, a simples introdução da tecnologia biomédica sem realizar modificações sociais, econômicas $e$ culturais acarreta efeitos apenas mínimos, se tanto, nos principais problemas de saúde enfrentados pela população. Ou seja, há fatores externos à Medicina, especialmente quando se trata de populações maiores, que devem ser considerados.

Grandes migrações populacionais nas últimas décadas têm forçado análises mais profundas das questões relativas à cultura. Isso tem sido especialmente verdadeiro nos Estados Unidos e na Europa, onde significativos contingentes de imigrantes já instalados começaram a procurar atenção médica e nem sempre obtiveram sucesso com os "procedimentos padrão" e guidelines empregados. Nesses casos, os confrontos entre culturas muito diversas chamam mais a atenção, ocorrendo em todas as dimensões do convívio social, sobretudo na área do cuidado à saúde, na qual a percepção das diferenças nas concepções de saúde/doença são cruciais para o resultado favorável do cuidado médico. 
$\mathrm{Na}$ esteira desse fenômeno, vem ganhando notoriedade a proposta de serviços de saúde que sejam culturalmente sensíveis, no sentido de qualificar profissionais de saúde e instituições para entender e satisfazer as necessidades próprias de saúde dos pacientes em locais em que essa diversidade cultural é mais pronunciada. Isto é, os serviços preparam-se para melhor acolher os pacientes que apresentam demandas de saúde bem específicas (Johnson \& Baboila, 1996; Hamilton, 1996; Shapiro \& Lenahan, 1996).

Contudo, acreditamos que não precisamos ir tão longe e esperar que venhamos a ter um grande volume de imigrantes estrangeiros para adequar os nossos serviços de saúde. A diversidade cultural no nosso país existe por todos os lados: há culturas brasileiras e mesmo culturas regionais bem estabelecidas (Oliven, 1992). Esse fato deve ser contemplado por nós, no dia-a-dia dos serviços de saúde. Isso exige uma postura que, em primeiro lugar, reconheça essas diferenças e, em segundo lugar, as aceite como parte indissociável do indivíduo, sem preconceitos e hierarquizações (Fonseca, 1995). Ao fazermos isso, inevitavelmente estaremos resgatando a tão falada e desejada cidadania e a democracia nos serviços de saúde, o que é um passo enorme na direção da consolidação efetiva do Sistema único de Saúde em nosso país.

Precisamos definitivamente entender que fatores culturais desempenham um papel crítico na prática de saúde em todos os âmbitos, inclusive no sistema formal de prestação de serviços médicos, entre nós fortemente assentado no modelo biomédico, o qual é apenas um entre tantos outros modelos.

Sem perder a perspectiva da contribuição que podemos dar à sociedade no sentido de oferecer atenção à saúde de alta qualidade, devemos estar capacitados para ouvir o paciente, perceber essas diferenças culturais $e$ adaptar nossa prática, dando margem a negociações com ele. Isso também significa qualidade! Esta busca não é nova, mas a atualidade oferece desafios ainda maiores, tanto pela diversidade cultural como pela complexificação crescente da nossa prática clínica. Assim, além de garantir competência na sua área técnica, os profissionais de saúde devem ser igualmente competentes para reconhecer as diferenças internas em cada subgrupo que compõe a nossa sociedade. E essa competência vai além da simples "sensibilidade" (Lavizzo-Mourey \& Mackenzie, 1995). Ou seja, o reconhecimento das diferenças culturais existentes em relação ao indivíduo que se encontra no nosso consultório deve servir de instrumento para que nós o auxiliemos a resolver seus problemas de saúde da melhor maneira possível. E esse tipo de postura deve ser estendida a todos os campos da atenção médica.

Isso tem a ver com a própria integralidade e humanização do cuidado de saúde, princípios dos mais caros à Medicina. E nessa abordagem integral e humana, a cultura é elemento essencial. É ela que influencia a forma como o indivíduo se percebe doente, leva-o a agir segundo alguns parâmetros bem definidos, a procurar tratamento $e$ a avaliar o que recebe (Lewis $e$ Williamson, 1995). Se não levarmos isso em conta, nossa abordagem será apenas parcial $e$, portanto, terá menor chance de alcançar o sucesso 
esperado.

Ouve-se com facilidade na área da saúde que "cada paciente é diferente". E nos darmos conta dessa diferença é que faz a prática médica tão fascinante. Os pacientes que nos chegam estão envolvidos por sua cultura como se esta fosse um manto e permanecem conectados a ela. Isso ocorre de modo semelhante à forma como estamos ligados ao conhecimento médico, pois no final das contas, nosso modelo biomédico também constitui um sistema cultural bem interessante de ser observado, mas que infelizmente é pouco questionado durante a formação dos nossos técnicos.

Seria muito bom ver os serviços de saúde e seus profissionais comunicando-se com seus usuários e perceber que por trás de cada paciente há uma cultura que dá sustentação à percepção que ele tem de sua doença $e$ do sistema de saúde.

O objetivo não é sufocar as outras formas de tratamento e de cura, mas justamente o contrário: entendê-las e valorizá-las no contexto em que se desenvolvem. Tudo em benefício dos pacientes e a partir deles.

O importante é deixar claro que o encontro de culturas está presente sempre quando um indivíduo procura alívio para os seus problemas de saúde. E que esse encontro é contínuo e dinâmico. Não começa e nem termina no encontro físico no consultório médico, por exemplo.

Referências

ATKINSON, S. Anthropology in research on the quality of health services. Cad. Saúde Públ., v.9, n.3, p.283-99, 1993.

BERGER, P., LUCKMANN, T. A construção social da realidade: tratado de sociologia do conhecimento. 12 ed. Petrópolis: Vozes, 1995.

BODSTEIN, R. Assistência médica na agenda pública. In: BODSTEIN, R (org.) Serviços locais de saúde: construção de atores e políticas. Rio de Janeiro: Relume-Dumará, 1995, p.15-39.

BOLTANSKI, L. As classes sociais e o corpo. Rio de Janeiro: Graal, 1984.

CALNAN, M. Towards a conceptual framework of lay evaluation of health care. Soc. Sci. Med., v.27, n.9, p. 927-33, 1988.

CAMARGO JR, K. Medicina, medicalização e produção simbólica. In: PITTA, A.M. Saúde \&

Comunicação: visibilidades e silêncios. São Paulo: Hucitec/ABRASCO, 1995, p.13-24.

FONSECA, C. Caminhos da adoção. São Paulo: Cortez, 1995.

FOUCAULT, M. Microfísica do poder. Rio de Janeiro: Graal, 1979.

GEERTZ, C. A interpretação das culturas. Rio de Janeiro: Zahar, 1978.

HAHN, R., KLEINMAN, A. Biomedical practice and anthropological theory: frameworks and directions.

Ann. Rev. Anthropol., v.12, p. 305-33, 1983.

HAMILTON, J. Multicultural health care requires adjustments by doctors and patients. Can. Med.

Assoc. J., v.155, n.5, p. 585-7, 1996.

HELMAN, C. G. Culture, health and illness: introduction for health professionals. 3. ed. Oxford: Butterworth-Heinemann, 1994.

JOHNSON, A., BABOILA, G. Integrating culture and healing: meeting the health care needs of a multicultural community. Minnesota Med., v.79, n.5, p.41-5, 1996.

KLEINMAN, A., EISENBERG, L., GOOD, B. Culture, illness and care: clinical lessons from anthropologic and cross-cultural research. Ann. Int. Med., v.88, n.2, p.251-8, 1978.

KLEINMAN, A. Patients and healers in the context of culture. Berkeley: University of California 
Press, 1980.

KLEINMAN, A. Social origins of distress and disease: depression, neurasthenia and pain in modern China. New Haven: Yale University Press, 1986.

KNAUTH, D. Os caminhos da cura: sistema de representações e práticas sociais sobre doença e cura em uma vila de classes populares. Porto Alegre, 1991. Dissertação (Mestrado) Universidade Federal do Rio Grande do Sul.

LANGMAN, J. Embriologia médica: desenvolvimento humano normal e anormal. 3.ed. São Paulo: Atheneu, 1977.

LAVIZZO-MOUREY, R, MACKENZIE, E. Cultural competence - an essential hybrid for delivering high quality care in the 1990's and beyond. Transactions of the American Clinical and Climatological Association, 1995. p.226-37.

LEWIS, JR., WILLIAMSON, V. Examining patient perceptions of quality care in general practice: comparison of quantitative and qualitative methods. Br. J. Gen. Pract., v.45, n.394, p.249-53, 1995.

LUZ, M.T. As instituições médicas no Brasil: instituição e estratégias de hegemonia. Rio de Janeiro: Graal, 1979.

OLIVEN, R.G. A parte e o todo: a diversidade cultural no Brasil-Nação. Petrópolis: Vozes, 1992.

PEDERSEN, D., BARUFFATI, V. Healers, deities, saints and doctors: elements for the analysis of medical systems. Soc. Sci. Med., v.29, n.4, p.487-96, 1989.

RHODES, L.A. Studying biomedicine as a cultural system. In: SARGENT, C., JOHNSON, T. Medical anthropology: contemporary theory and method. Westport: Praeger, 1996, p.165-80.

SHAPIRO, J., LENAHAN, P. Family medicine in a culturally diverse world: a solution-oriented approach to common cross-cultural problems in medical encounters. Fam. Med., v.28, n.4, p.249-55, 1996.

WILLIAMS, S.J., CALNAN, M. Modern medicine and the lay populace: theoretical perspectives and methodological issues. In: WILLIAMS, S.J., CALNAN, M. (eds.) Modern medicine: lay perspectives and experiences. London: University College London, 1996. p.2-25.
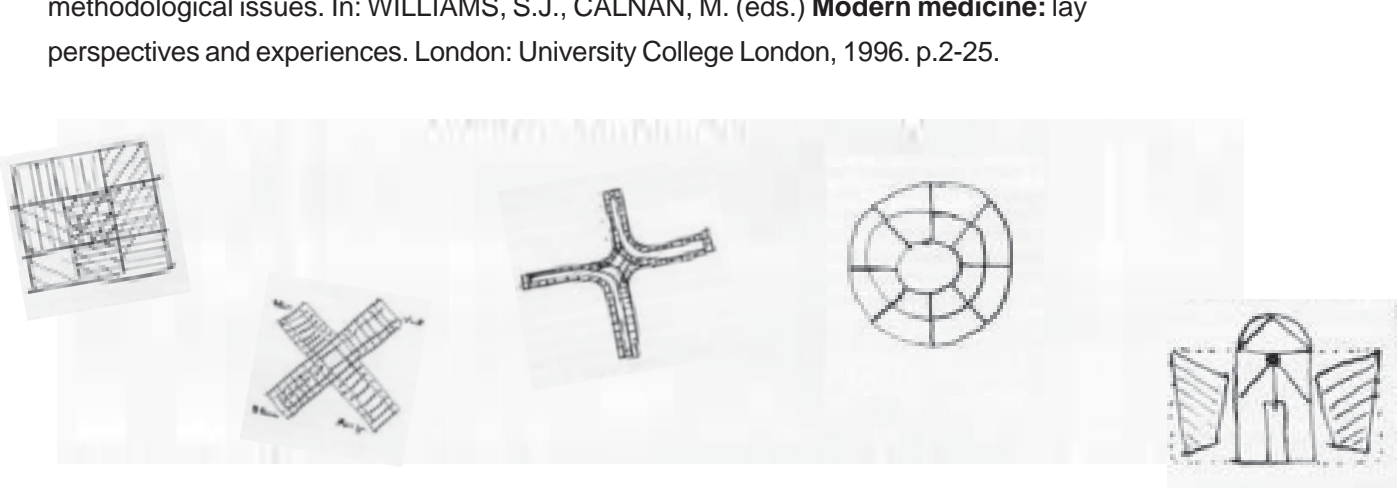

OLIVEIRA, F. A. Antropología en los servicios de salud: integralidad, cultura y comunicación, Interface _ Comunic, Saúde, Educ, v.6, n.10, p.63-74, 2002.

La utilización de otras áreas del conocimiento en el campo de la salud ha sido intensificada en los últimos años. Las ciencias sociales de modo general y la antropología médica, específicamente, han contribuido, de manera decisiva, para la comprensión de los fenómenos relacionados con el proceso salud/enfermedad, tanto individual como colectivamente. En este artículo, el autor expone una serie de consideraciones buscando un mejor entendimiento para el tema de la atención a la salud, con énfasis en la aclaración de las formas de interacción y comunicación entre esos servicios de salud y sus usuarios. El artículo termina demostrando como el hecho de centrar la práctica médica en el usuario y en su cultura, acaba beneficiando a pacientes y profesionales, en el sentido de recobrar la humanización del cuidado y de la integralidad de la asistencia a la salud.

PALABRAS CLAVE: Antropología médica; servicios de salud; asistencia a la salud. 